\title{
An Energy Efficient QoS Routing Protocol based on Red Deer Algorithm in MANET
}

\section{S. Arivarasan ${ }^{a}$, Dr.S. Prakash ${ }^{b}$, Dr.S. Surendran ${ }^{c}$}

${ }^{\text {a}}$ Research Scholar, Sathyabama Institute of Science and Technology, Chennai, Tamil Nadu, India. E-mail: arivarasan.selvam@gmail.com

${ }^{\text {b} P r o f e s s o r, ~ D e p a r t m e n t ~ o f ~ E l e c t r o n i c s ~ a n d ~ C o m m u n i c a t i o n ~ E n g i n e e r i n g, ~ S a v e e t h a ~ S c h o o l ~ o f ~ E n g i n e e r i n g, ~}$ SIMATS, Thandalam, Chennai, Tamil Nadu, India. E-mail: prakash.sav4@gmail.com

'Professor \& Head, Department of Computer Science and Engineering, Tagore Engineering College, Tamil Nadu, Chennai, India. E-mail: babume74@gmail.com

Article History: Received: 11 January 2021; Accepted: 27 February 2021; Published online: 5 April 2021

\begin{abstract}
MANETs (Mobile ad hoc networks) are overloaded with their heavy usage choking traffic. Recent studies have been attempting to find feasibility of routes in these networks. Most proposed schemes have failed to consider the network traffic or MANET requirements. MANET routing done by RPs (Routing Protocols) would perform better when they adhere to QoS (Quality of Service) or application requirements. As a result, RPs must satisfy numerous QoS metrics at a time where soft computing algorithm based RPs are a solution to QoS routing problems. Evolutionary algorithm based routing do not achieve enough energy efficiency which is a major drawback. To overcome this drawback, this work, proposes Red Deer algorithm (RDA) based energy efficient QoS routing for MANETs called (RDA-EQRP). The proposed RDA based routing algorithm discovers the shortest path from a source to a destination which can consumes less energy while supporting the metrics such of reliability, bandwidth, SRC (Static Resource Capacity), Quality and delay. Simulation results show that proposed rotting scheme RDA-EQRP consumes lesser energy in MANET routing.
\end{abstract}

Keywords: Energy Efficient QoS Routing, MANET, Red Deer Algorithm, RDA-EQRP, QDAS, GA-QMR and CSO-AODV.

\section{Introduction}

MANETs are infrastructure and self-organized wireless networks and are not controlled centrally [1]. MANET routing involves optimal paths in routing while following strict compliance to QoS parameters like bandwidths or delays in real time environments. Multimedia application which consume heavy amounts of bandwidths need to follow stringent QoS requirements. MANETs can guarantee quality in its services when they deliver on minimal bandwidths and minimum delays. One major issue in MANETs is handling link failures and recovering its routes which occur due to the mobility of nodes. MANET's performance is directly affected by its efficiency to recover from changes in its links.

MANETs offer many multimedia facilities based on videos like video conferencing or video-on-demand. The burden directly shifts to RPs which has to manage paths when these nodes are mobile. Most MANET RPs do not consider QoS problem though QoS helps increase the network's performance. Multimedia applications which comply strict QoS requirements, achieve in delivering packets better. Thus, the goal of QoS is achieving a stable and deterministic behavior in MANETs where packets are delivered reliably. However, QoS implementations are challenged by node's mobility in MANETs. RPs which caters to QoS in the network is found to be unsatisfactory or impractical when they involve heavy computational overheads. QoS based routing need to maintain the network's up-to-date node state information while finding possible paths for a connection in the network.

It is compulsory for RPs to take into account battery energy, available bandwidths and end-to-end delays for satisfying QoS in a network. These parameters become main factors while designing a RP for MANETs with QoS. Hence, the QoS parameters considered in this study include delay times, bandwidths and energy consumptions of nodes in its proposed RDA-EQRP routing for MANETs. The MANET routes in this study uses additional QoS parameters of reliability, quality and SRC. The study's proposed fitness function includes the listed parameters for implementations. Thus, this paper's contributions can be summarized to a new routing protocol for MANETs which caters to six quality parameters for efficient routing in the network. The section that follows includes a review of literature. The proposed RP's methodology is detailed in sections 3 and 4 while its results are presented in section 5. The final section concludes this paper.

\section{Related Work}

QoS-sensitive RP for multiple paths for multimedia content was proposed in [2]. The scheme based on ACO (Ant Colony Optimization) established multiple paths for routing for enhancing network reliability. QoS was included with ACO in [3]. The proposed QAMR foraged ant's behaviours in its path selections and data transmissions. The scheme used node stability and probabilities of paths in its route selections. 
PSO (Particle Swarm Optimization) was used in MANET node's lifetime predictions and routes were discovered in the study [4]. The technique computed lifetime and bandwidths of links based on a node's relative mobility and energy usage. The study in [5] maintained QoS while resolving a sub optimal solution for its routing. The scheme called GA-QR used GA (Genetic Algorithm) and quality in routing by implementing clustering preferences in GA evolutions and stipulations.

Hybrid optimization was used for MANET routing in [6]. The scheme called M-LionWhale implemented LA (Lion Algorithm) combined with WOA (Whale Optimisation Algorithm) for its optimized path selections MANETs. Their multi-objective optimisations took into account the QoS parameters of distances, energies, trust, delays and link lifetimes.

Multi-cast routing was proposed in [7] which mimicked multi-objective bacteria for their multi-casts. The scheme called RBMOBFO was based on referential user preferences. The algorithm considered user inputs as reference points in a problematic search space. ACO based RPs in MANETS have issues in balancing loads and using their energy efficiently. The study [8] proposed A-EEBLR selection of the next hop node based on energy usage, delays, and link quality and traffic congestions.

\section{Proposed Methodology}

MANETS are made of battery operated devices that exchange information using a wireless medium. The devices need to conserve their battery energy to stay longer in the network. These minimizations involve energy reductions during transmissions of requests. The basic communications are done by RPs which needs to be energy efficient which can be achieved by distributing transmission energy equally amongst nodes. Thus, node energies play an important role in MANET information routing. This research work proposes a MANET protocol that checks the network's node energy levels prior to its routing while ensuring its pre-defined QoS parameters are satisfied. Figure 1 depicts the overall flow of the proposed RDA-EQRP technique's path selection in MANETs.

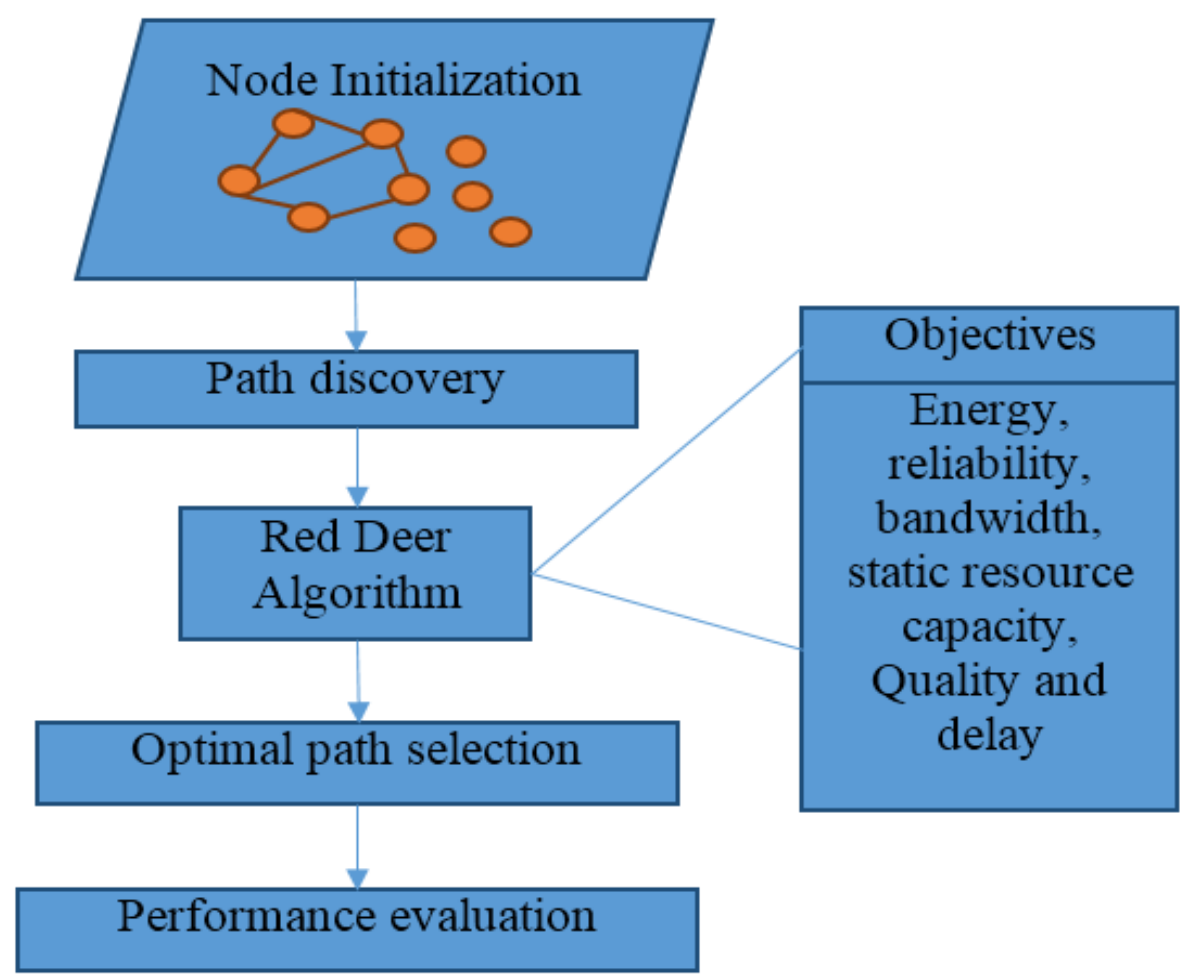

Fig. 1. RDA-EQRP Path Selection

The persistent nodes in MANETs are using by RDA for routing information where optimized paths are selected using a fitness function. The function evaluates paths based on the parameters of reliability, energy, bandwidth, delay, quality and SRC. When several paths are discovered for routing, the path using minimum or shortest energy are selected. Deficiencies in RDA based routing are overcome in this work by using several QoS parameters as a 
part of the fitness function. This work also accommodates the mobility of nodes or their dynamic nature while assessing network performances. The implementation of the proposed method minimizes QoS on sourcedestination links while satisfying QoS constraints for overcoming MCP (Multi-Constrained Path) issues. The proposed work's phases include initializations, discovery and optimizations in path selections.

\subsection{Defining the Network}

Assuming a network as a graph $G=(V, E), \mathrm{V}$ represents the set of all nodes and the set of all links is $E$ is the set of all links. If $r$ stands for the transmission range and, the distance between two adjacent nodes and $d \leq r$ then the link $e(e \in E)$ exits and is a two-way link between. $P$ is the set of paths from a source (s) to a destination (D) then $(s, D \in V) . E(p)$ is the set of Edges and $N(p)$ is the set of all nodes for a path $p(p \in P)$. QoS of a path on $T$ from $s$ to a destination $d$ is denoted as $T C$. Every link is represented as the cost of its reliability, energy, bandwidth, delay, quality and SRC. The MANET nodes in a dynamic environment are primarily initialized where MNs (Mobile Nodes) act as hosts and routers. The links between MNs is used to create the MANET. MNs computed co-ordinates help in using its location or velocity and If $m$ is the set of nodes then $1<\mathrm{i}<\mathrm{m}$. The next step, path discovery is based on available links in nodes for communication. If $\mathrm{P}$ stands for paths present between a source and a destination node, then it can be said that $1<\mathrm{j}<\mathrm{P}$.

\subsection{QoS based optimized RDA Routing with QOS}

QoS parameters are a part of the cost function in this proposed model both for optimizations and minimizing practical constraints in routing. Constraints of reliability, Energy, bandwidth, delay, quality and SRC are included in the optimization model.

Reliability: This refers to the stability or dependability of a network in cases of singular transmissions from a path $p$ to the sink, the reliability of the path can be depicted as Equation (1).

$$
R_{p}=\prod_{l=1}^{\text {hop p }_{p}} R_{\text {link }_{l}}
$$

Where, $R_{\text {link }}$ - link reliability and $l$ - available links count.

Energy: Mobile nodes deplete their energies faster. Efficient use of battery energy helps in improving the efficiency of the network as its total lifetime is dependent of the associated node energies. Energy can be computed using Equation (2).

$$
R^{\text {energy }}=\frac{1}{m n * p} \sum_{i=1}^{m n} \sum_{\substack{j=1 \\ i \in j}}^{P} E n_{i j}
$$

Where, $1<i<m n$ - network's mobile nodes, $1<j<p$ - paths count, $E n_{i j}$ - node energy in a path. The energy value is computed using Equation (3).

$$
\text { energy } E n_{i j}=P_{T p}^{i} * E n_{T X}^{i}+P_{R p}^{j} * E n_{R X}^{j}
$$

and $E n_{T p}^{i}=T p_{t}^{i}-T X_{p}^{i}, E n_{R p}^{j}=R p_{t}^{j}-R p_{p}^{j}$

Where, $T p$ - transmission power, $R p$ - receiver's power and $P$ - size of the packet.

Bandwidth: MANET's bandwidth, an important factor required for quick or effective transmissions can be obtained by using Equation (4).

$$
\operatorname{BandWidth}(R(s, d))=\min (b w(s)), s \in R(s, d)
$$


Delay: Delays in end relays does affect the transmissions and longer delays implies more energy (as depicted in (2) is consumed. Delay values are obtained using Equation (5).

$$
\text { delay }=\sum_{N=0}^{n} \frac{(\text { received time }- \text { sent time })}{N}
$$

Quality: Equation (6) is used in this work to compute link quality between nodes and its 1-hop neighbors:

$$
\text { quality }=\frac{\text { M1hopRe }}{\text { TM1hopSent }}
$$

Where, $M 1$ hopRec - Hello message received from 1 hop neighbour in node $N$; TM1 hopSent - Total Hello message sent by 1-hops.

SRC: SRC for a node is computed using packet queues $P_{q}(M B)$, CPU speed CPU $P_{C P U}(G H z)$, Battery power battery $M_{b}(m W)$, and maximum available bandwidth BandWidth (kbps) and depicted in Equation (7).

$$
S R C=\gamma * P_{q}+\lambda * P_{C P U}+\beta * M_{b}+\alpha * \text { BandWidth }
$$

Where $\gamma, \lambda, \beta$, and $\alpha$-node characteristics that are weighed with their sum $=1$.

Fitness Function: The function $F(x)$ for the proposed RDA-EQRP is computed using Equation (8).

$$
F(x) \rightarrow \text { Fitness }=\frac{1}{p} \sum_{i=0}^{p} T C_{i}
$$

Where, $T C_{i^{-}}$cumulative QoS on route $i$ and $p$ - route paths suggested by the proposed function. Cumulative QoS includes all the pre-defined parameters of the proposed technique. Each parameter taken into consideration is assigned a weight like $\zeta, \alpha, \beta, \lambda, \delta$ and $\varepsilon$ which are threshold values. These weights are computed collectively using Equation (9):

$$
T C=\zeta * \text { energy }+\alpha * \text { delay }+\beta * \text { quality }+\lambda * R_{p}+\delta * S R C+\varepsilon * \text { BandWidth }
$$

\subsection{RDA Path Discovery}

Initialization in the study by randomizing the population, RDs (Red Deers). The number optimal RDs are termed "male RD" while balance nodes are termed "hinds". Male RDs roar based on their capacity which is classified into Commander roars and Stag roars. Commanders then build harems which is the count of hinds and is dependent on the commander's roaring strength. Commanders mate with hinds in a harem and stags mate with nearby hinds without limitations on harems [11]. The flowchart of RDA route discovery is depicted in Figure 2. RDA uses mathematical formulations for its minimizations where three significant attributes manage explorations. $\alpha$ and $\beta$ supervise diversification phases while $\gamma$ balances intensifications. All these parameters range between 0 and 1 . 


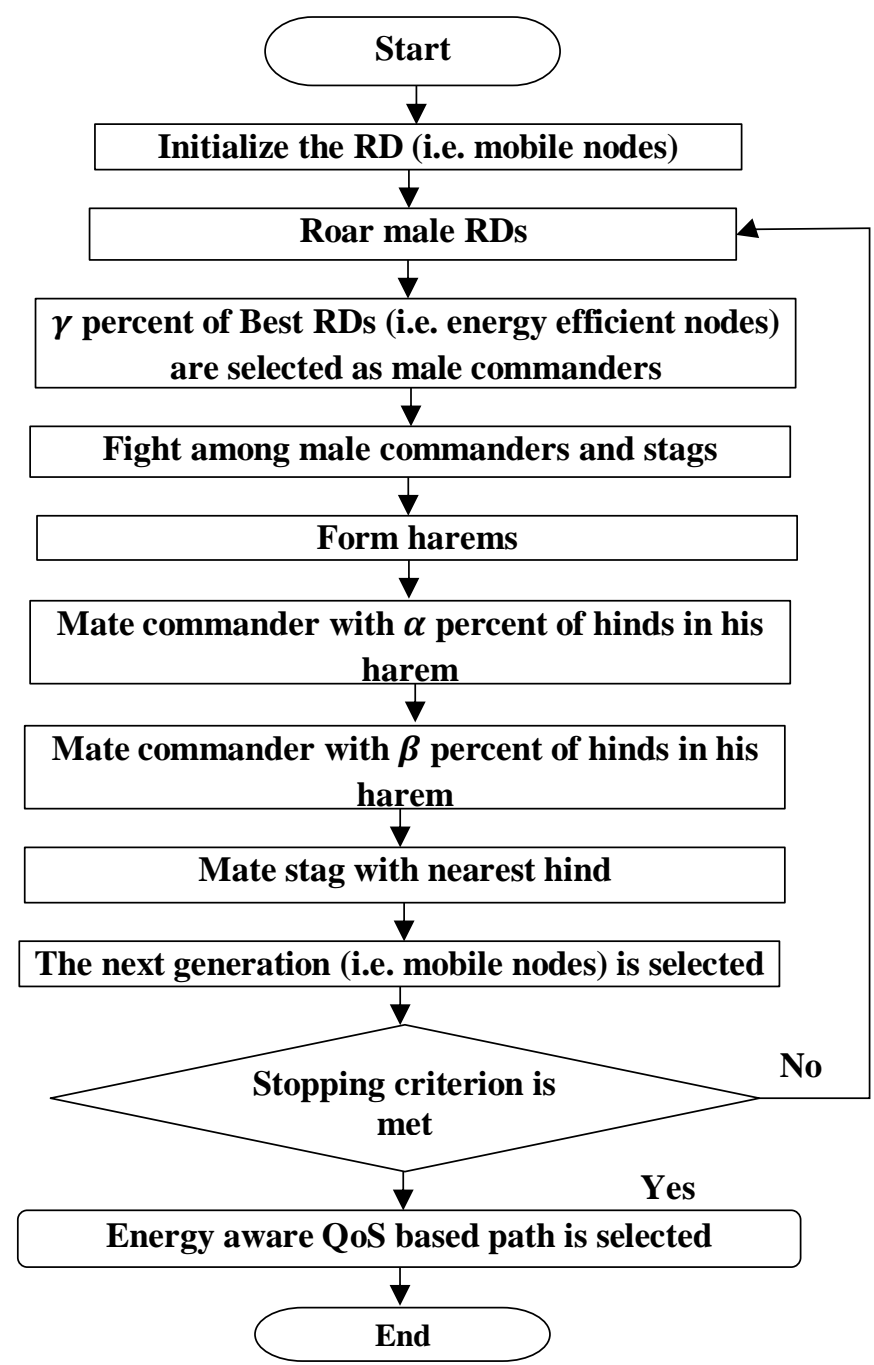

Fig. 2. RDA route selection flowchart

Generating primary RDs: The main purpose of optimization methods is in identifying a near-optimal solution from given variables. Array's in GA are termed "chromosomes", but "Red Deer" in RDA which provide possible solution $\mathrm{S}$ in the solution space with dimensionality $N_{v}$. The optimal path selection in RD is a $1 \times N_{v}$ array which can formulated using Equation (10).

$$
R D=\left[S_{1}, S_{2}, \ldots, S_{N_{v}}\right]
$$

The function for estimating all RDs is given in Equation (11).

$$
V=f(R D)=f\left(S_{1}, S_{2}, \ldots, S_{N_{v}}\right)
$$

The initial population of mobile nodes are sized $M N_{\text {pop }}$ from which optimal RDs $M N_{\text {male }}$ and $M N_{\text {hind }}\left(M N_{\text {hind }}=M N_{\text {pop }}-M N_{\text {male }}\right)$ are chosen. Thus, $M N_{\text {male }}$ count depicts the elated condition of MANETs or $M N_{\text {male }}$ count retains nodes satisfying QoS constrains and $M N_{\text {hind }}$ vary in this phase.

Male RD's roaring intensity: Nodes (RDs) improve their efficiency with their roars which might be effective or ineffective. Male RDs depict optimal solutions in the solution space where RDs modify their location for upgrading the places of males as given by Equation (12).

$$
\text { male }_{n e w}=\left\{\begin{array}{l}
\text { male }_{01 d}+x_{1} \times\left((u b-l b) * x_{2}\right)+\text { lb if } x_{3} \geq 0.5 \\
\text { male }_{01 d}-x_{1} \times\left((u b-l b) * x_{2}\right)+l b \text { if } x_{3}<0.5
\end{array}\right.
$$


Solution found in male RDs is limited by upper and lower boundaries. male ${ }_{01 d}$ Refers to RDs current location while male $_{n e w}$ represents its upgraded place. Randomized roaring processes, $x_{1}, x_{2}$ and $x_{3}$ are produced in a uniform distribution range between 0 and 1 . RDs extend their territory randomly by roaring. Thiis impacts solutions where M, a novel location is approved with its objective fitness which should be better than the previous solution where $y$-axis has the objective function values for locations in the $x$-axis.

Selecting best RD commander with percent: male RDs vary in their energy levels, appearances and expansions and are treated as commanders or stags. Commander counts are determined using Equation (13).

$$
M N_{c}=\operatorname{round}\left\{\gamma \cdot M N_{\text {male }}\right\}
$$

where $M N_{c}$ - count of males. $\gamma$ value is defined with an initial value between 0 and 1 . Equation (14) estimates male stags in the herd.

$$
M N_{\text {stag }}=M N_{\text {male }}-M N_{c}
$$

where $M N_{\text {stag }}$ - stags in the male population.

Fight between male commanders and stags: Assuming commanders fight with stags randomly, it is possible to get two novel solutions. This solution can be interchanged by the commander for an optimized solution which is a better solution in four offered solutions namely commander, stag, and 2 novel solutions. The fights can be depicted mathematically as Equations (15) and (16).

$$
\begin{aligned}
& S_{\text {new } 1}=\frac{(\text { comd }+ \text { stag })}{2}+y_{1} \times\left((u b-l b) * y_{2}\right)+l b \\
& S_{\text {new } 2}=\frac{(\text { comd }+ \text { stag })}{2}-y_{1} \times\left((u b-l b) * y_{2}\right)+l b
\end{aligned}
$$

where $S_{\text {new } 1}, S_{\text {new } 2^{-}}$Novel solutions produced in fights. The upper and lower bounds limit the search space resulting in a uniform distribution of solutions in the range 0 to 1 from fights y1 and y2 and allocated by the objective function. High energy node becomes the winner while the node with low energy loses the contest. The objective function decides on $S_{n e w 1}$ as the latest commander based on its results.

Harems population count: The energy level of the male commander is the deciding factor for the number hinds that belong to a harem. Objective function decides the effectiveness of a commander using Equation (17):

$$
t_{n}=e_{n}-\max \left\{e_{i}\right\}
$$

where $e_{n}$ - energy of nth commander and $t_{n}$ depicts - normalized value. The estimation of normalized power of commanders is computed using Equation (18).

$$
P w_{n}=\left|\frac{e_{n}}{\sum_{i=1}^{M N_{c}} e_{i}}\right|
$$

This normalized energy can also be defined in terms of the hinds count which is evaluated using Equation (19):

$$
M N \cdot \text { harem }_{n}=\operatorname{round}\left\{P w_{n} \cdot M N_{\text {hind }}\right\}
$$

Where, $M N$. harem $_{n}$ - number of hinds in nth harem and $M N_{\text {hind }}$ - total hinds. $M N$. harem ${ }_{n}$ is selected randomly from hinds for classifications. Thus, the commander with optimal fitness value will obtain maximum number of hinds.

Commander mates in a harem with $\boldsymbol{\alpha}$ percent: All living beings mate by nature for producing an offspring or new generation. The commander mates with $\alpha$ number of hinds in the harem for his mating and represented as Equation (20). 


$$
M N \cdot \text { harem }_{n}^{\text {mate }}=\operatorname{round}\left\{\alpha \cdot M N \cdot \text { harem }_{n}\right\}
$$

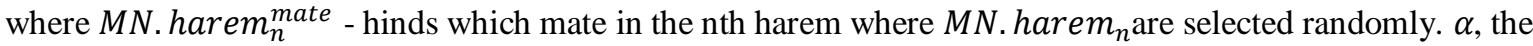
initial parameter of RDA has to be in the ranger $((0,1)$. Mating is determined using Equation $(21)$ :

$$
N S=\left(\frac{\text { comd }+ \text { hind }}{2}\right)+(u b-l b) \times z
$$

where Comd - commanders and Hind - hinds. NSs, defined as a new solution where z's value is arbitrary in the range 0 and 1.

percent of hinds in another harem: The male commander also mates with $\beta$ number of hinds in randomized fashion which is done for expanding his territory. $\beta$ is also an initial parameter of this approach and the $\beta$ count can be obtained using Equation (22).

$$
M N \cdot \text { harem }_{k}^{\text {mate }}=\operatorname{round}\left\{\beta \cdot M N \cdot \text { harem }_{k}\right\}
$$

where $M N$. harem mate - value of hinds that mate with the commander in the kth harem.

Stag mating with its nearest hind: Stags identify nearest hinds for their mating in a harem by computing the distance between a stag and a hind in a -dimension using Equation (23):

$$
\operatorname{dis}_{i}=\left(\sum_{j \in J}\left(\operatorname{stag}_{j}-\operatorname{hind}_{j}^{i}\right)^{2}\right)
$$

Where, $d i s_{i}$ - distance from i-th hind/stag. A lower value in the matrix is the selecting criteria in case of hinds which prepares it for mating. This process is handled numerically as presented in Equation (21).

Selecting subsequent generation: Subsequent generation is selected based mainly on two principles. All male RDs are retained referring to commanders and stags. This implies that remaining population is the next generation. Thus, the hinds and offspring produced are selected on the basis of a fitness value obtained by the fitness function.

Stopping condition: The recursive operations are stopped once optimal paths are identified within a specific period as illustrated in Figure 3.

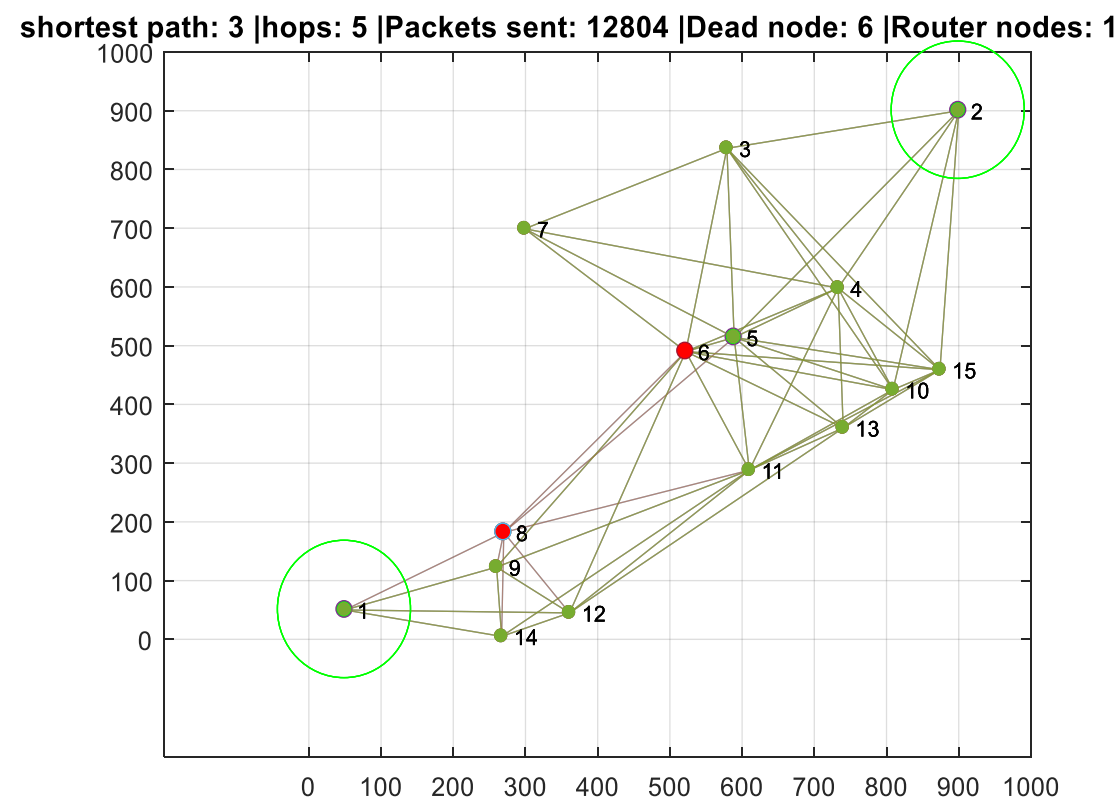

Fig. 3. RDA Iteration Stopping Conditions 
Algorithm 1. Red deer algorithm based optimal path selection

Input: Initialize the red deer population (i.e. mobile nodes), Energy, reliability, bandwidth, static resource capacity, Quality and delay

Output: energy efficient based node selection for optimal path

Calculate fitness $F(x)$, sort and form hinds $\left(M N_{\text {hind }}\right)$ male commanders $\left(M N_{\text {male }}\right)$

$$
\begin{gathered}
S^{*}=\text { the best solution } \\
T_{1}=\text { clock; }
\end{gathered}
$$

While ( $\mathrm{t}<\max$ simulation time)

for each male RD

Roar the male eq. (12)

Update current position if better than previous ones

end for

Sort population for finding stags and commanders from equations (13) and (14)

for each male RD

Tussle between male RDs and stags :Equations (15) and (16)

Update male RD and stag positions

end for

form harems using Equations (17-19)

for each male RD based on Equation (20)

randomly mate with selected harem hinds using Equation (21)

select a random harem k using Equation (22)

male RDs mate with selected harem hinds using Equation (21)

end for

foreach stag

find distance between stag and all hinds for selecting nearest hind by Equation (21)

mate the stag with selected hind using Equation (21)

end for

next generation selection

update $S^{*}$ if there is a better solution

end while

$$
\begin{aligned}
& T_{2}=\text { clock } ; \\
& t=T_{1}-T_{2}
\end{aligned}
$$

return $S^{*}$ as the best value of path selection

Repeat maximum paths found until stopping criterion is met.

Display energy aware optimal path with better QoS and end the process

\section{Results and Discussion}

This section displays obtained results of experiments. Seventy mobile nodes were randomly scattered in a 1000 $\times 1000$ square field. The nodes moved randomly based on waypoint model in speeds varying in the range $5-30 \mathrm{~m} / \mathrm{s}$ with a transmission range of $250 \mathrm{~m}$. Results of simulations on four metrics (Throughput, Delay, Detection Accuracy, Consumed Energy and Routing Overheads) were used to evaluate the proposed technique's performance. Throughput is the count of packets received at a destination node in the unit of time. Delay is the latency of a data bit in communication between endpoints. Detection Accuracy is the number of malicious nodes detected from the total count of malicious network nodes. Energy consumption is the energy used by nodes in transmissions. Routing Overheads is the count of routing packets received at a destination divided by the total data packet count. The proposed RDA-EQRP is also compared with DACO [12] and GA-QMR [10] techniques.

\subsection{Throughput (TP)}

Fig. 2 shows the comparison result of throughput from the proposed RDA-EQRP, and the existing DACO and GA-QMR methods. It is noted that the proposed EDTDS-AODV attains higher throughput rate of $0.95 \mathrm{kbps}$ when 
compared with all the other proposed and existing approaches such as DACO and GA-QMR. The performance of throughput by nodes is observed to be still higher for further increasing nodes too. The reason is that, the proposed work has the capable of identifying optimal nodes using RDA with routing table collection database. Also, RDAEQRP is designed to meet QoS requirement with less energy consumption. This boundary helps to protract the optimal solution availability for a longer time which leads the throughput would be higher.

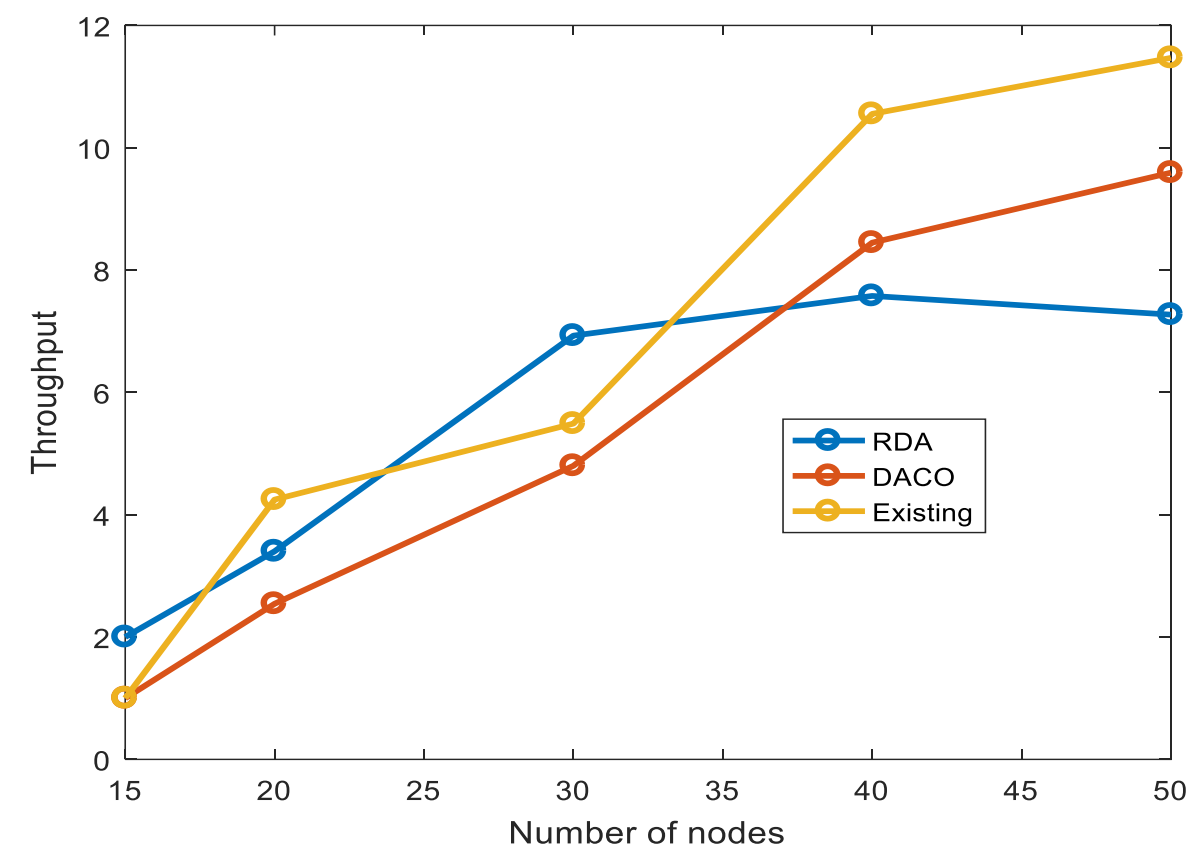

Fig. 2. Throughput vs. No of Nodes

\subsection{Delay}

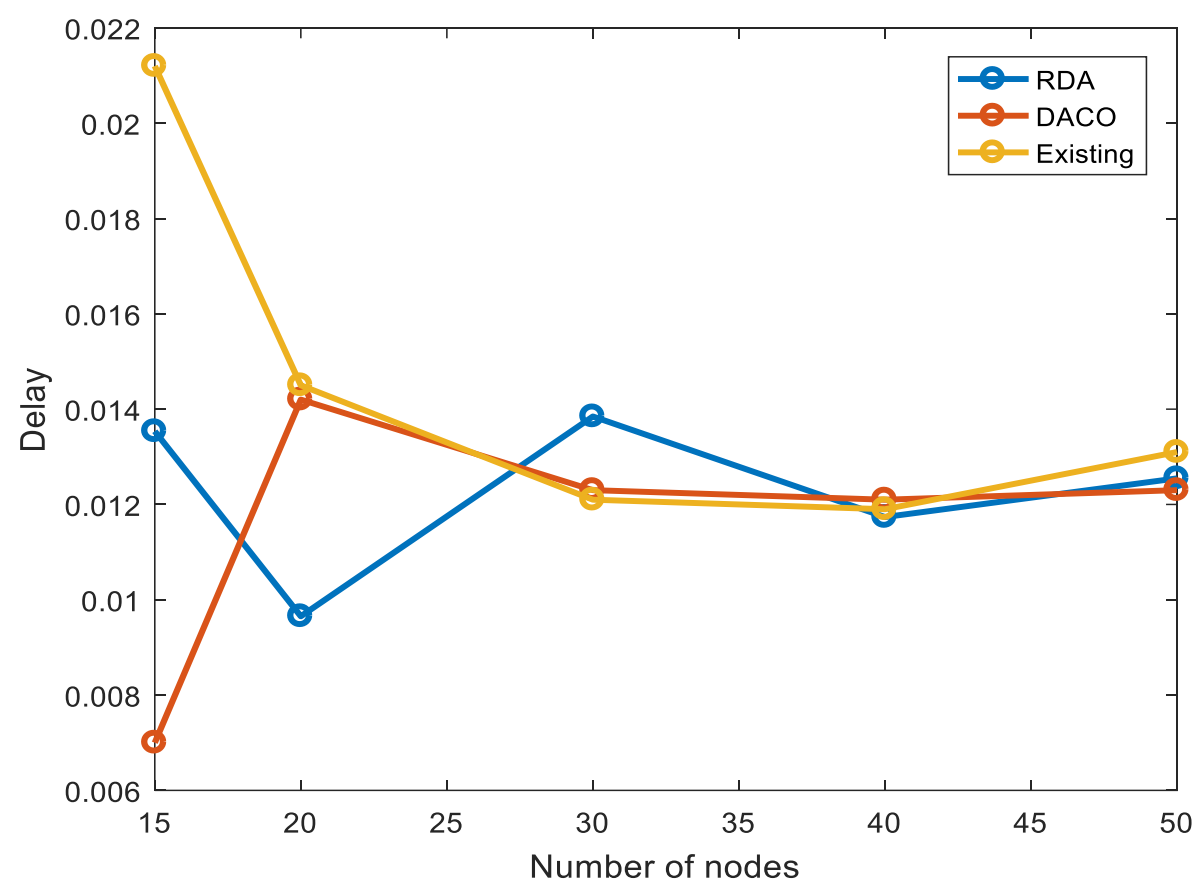

Fig. 4. Delay/ Nodes Count

Delay shown in Figure 4, projects the state of the message that is sent to a destination. RDA-EQRP has a higher ratio when compared to GA-QMR and DACO. The delay decreases as the number of nodes increases. RDA-EQRP has a lower delay rate, since it uses a stable path which is restored quickly on adverse conditions and without suspending transmission. This results in an increased PDR (Packet Delivery Ratio). 


\subsection{Detection Accuracy}

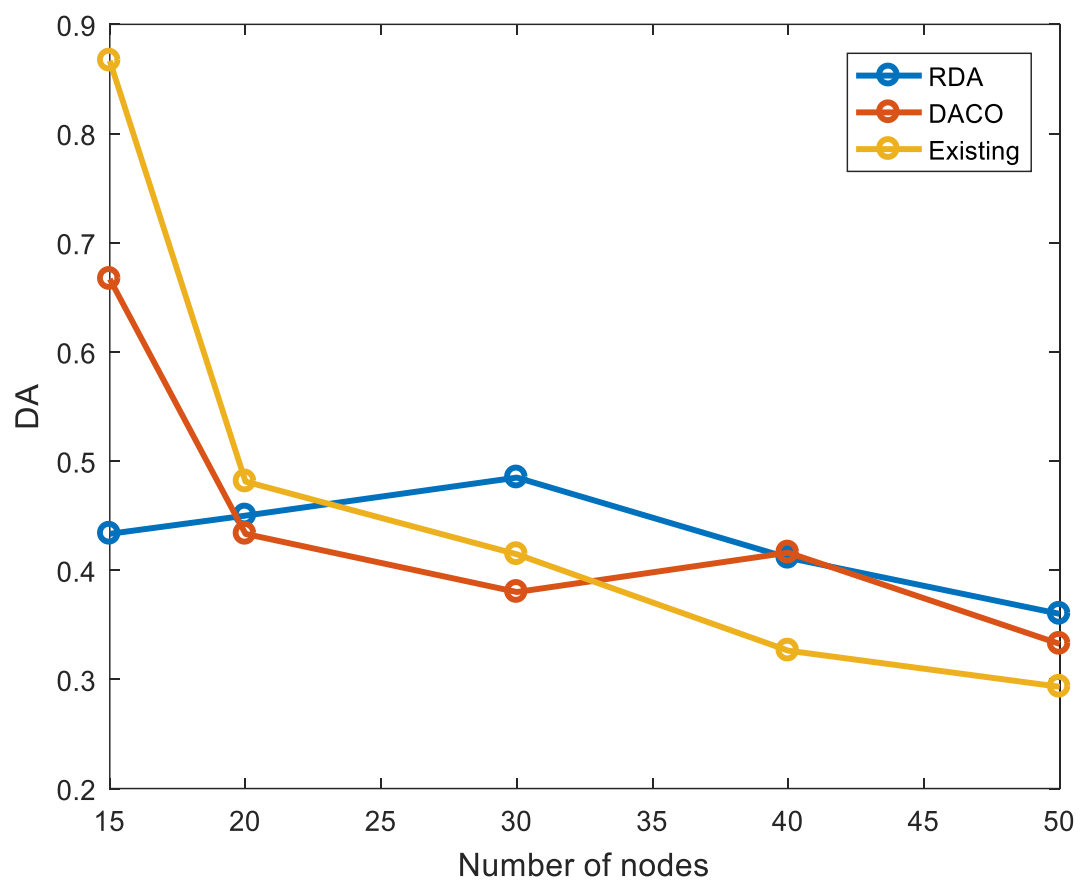

Fig. 5. Detection Accuracy/ Nodes Count

Detection of malicious nodes is depicted in Figure 5 with comparisons. RDA-EQRP achieves better accuracy in malicious node detection when compared to DACO and GA-QMR as its selections are based on RDs fitness evaluations of nodes holding minimum energy. The detection accuracy decreases as nodes count increases.

\subsection{Energy Consumption}

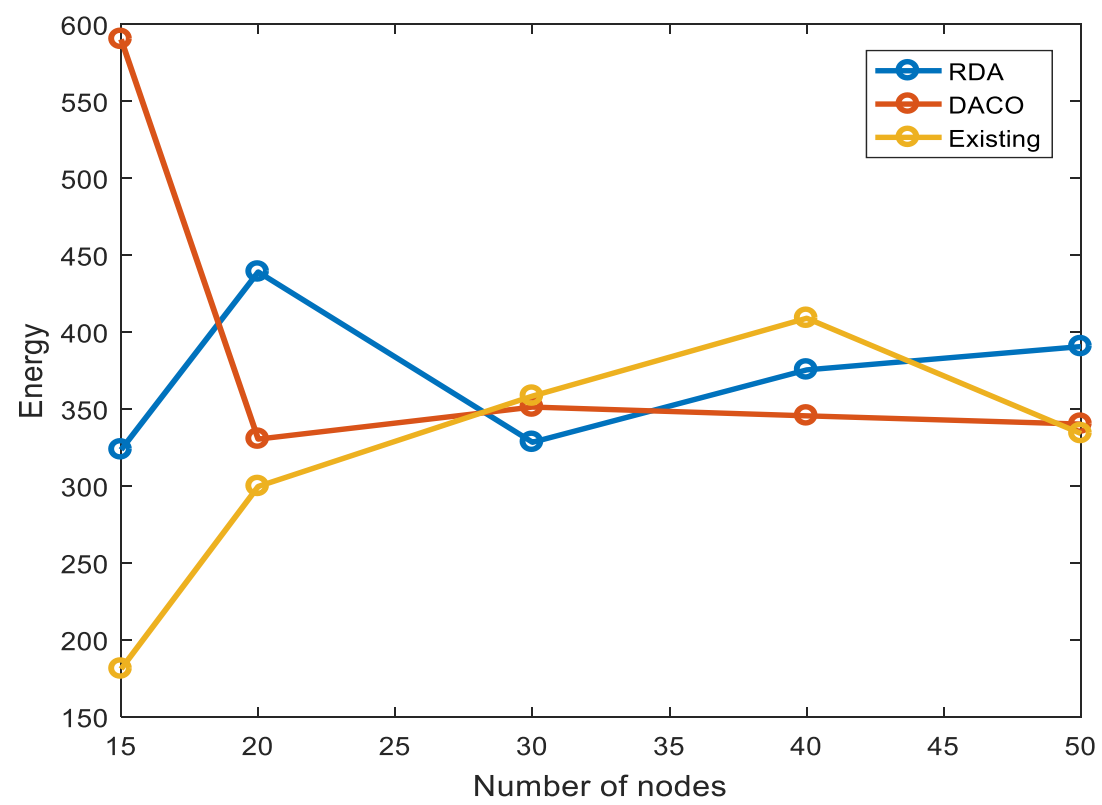

Fig. 6. Energy Consumption/Nodes Count

The energy consumption of RDA-EQRP is lesser than other methods in transmissions. Energies of nodes decrease due to malicious attacks. The proposed methods malicious node detection helps identify alternative routes thus increasing the node energy. Moreover the proposed RDA-EQRPR routing built on RDA ensures maximum stability in its pre-mature convergence and cost factors thus navigating the network towards minimized energy consumptions. 


\section{Conclusion and Future Work}

This work has proposed and demonstrated an energy based protocol, RDA-EQRP that identifies nodes that deplete their energies quickly in MANET transmissions. Unreliable nodes are eliminated from routing paths by the proposed technique, thus saving maximum node energies of the network. This proposal has also demonstrated the effective use of QoS in protocol implementations. RDA-EQRP provides improved quality by using RDA in its routing. Thus, RDA-EQRP can be implemented in other MANET routing protocols for checking their QoS related performance. Future scope of this work lies encrypting transmitted packets and provide virtual energy for source nodes in MANETs.

\section{References}

1. Kopekar, S., Kumar, A., Bhowmick, K., Shah, M., Patel, K.J., Raja, N.M., \& Harasani, W. (2015). A study of ad-hoc wireless networks: various issues in architectures and protocols. International Journal of Computer Applications, 122(6), 36-40.

2. Kim, S. (2012). An ant-based multipath routing algorithm for QoS aware mobile ad-hoc networks. Wireless Personal Communications, 66(4), 739-749.

3. Krishna, P.V., Saritha, V., Vedha, G., Bhiwal, A., \& Chawla, A.S. (2012). Quality-of-service-enabled ant colony-based multipath routing for mobile ad hoc networks. IET communications, 6(1), 76-83.

4. Manickavelu, D., \&Vaidyanathan, R.U. (2014). Particle swarm optimization (PSO)-based node and link lifetime prediction algorithm for route recovery in MANET. EURASIP Journal on Wireless Communications and Networking, 2014(1), 107.

5. Gurumoorthy, K.B., \& Kumar, A.N. (2018). Mutual constraint based GA suggested routing algorithm for improving QoS in clustered MANETS. Wireless Personal Communications, 98(3), 2975-2991.

6. Chintalapalli, R.M., \& Ananthula, V.R. (2018). M-Lion Whale: multi-objective optimisation model for secure routing in mobile ad-hoc network. IET Communications, 12(12), 1406-1415.

7. Sahoo, S.P., Nayak, S., \& Kabat, M.R. (2018). A reference-based multiobjective bacteria foraging optimization technique for QoS multicast routing. Arabian Journal for Science and Engineering, 43(12), 7457-7472.

8. Karmel, A., Vijayakumar, V., \& Kapilan, R. (2019). Ant-based efficient energy and balanced load routing approach for optimal path convergence in MANET. Wireless Networks, 1-13.

9. Singh, S., Koslia, M., \& Poonia, R.C. (2018). A GA-QMR: Genetic Algorithm Oriented MANET QoS Multicast Routing. Recent Patents on Computer Science, 11(4), 268-275.

10. Mandhare, V.V., Thool, V.R., \& Manthalkar, R.R. (2016). QoS Routing enhancement using metaheuristic approach in mobile ad-hoc network. Computer Networks, 110, 180-191.

11. Fathollahi-Fard, A.M., Hajiaghaei-Keshteli, M., \& Tavakkoli-Moghaddam, R. (2020). Red deer algorithm (RDA): a new nature-inspired meta-heuristic. Soft Computing, 1-29.

12. Sabri, N.A.M., Hussin, B., \& Samah, K.A.F.A. (2017). Dijkstra-ant colony optimization algorithm for shortest and safest evacuation in high rise building. Jurnal Teknologi, 79(3), 69-77. 